\title{
INGESTA DE ARSÉNICO: EL IMPACTO EN LA ALIMENTACIÓN Y LA SALUD HUMANA
}

\author{
María Medina-Pizzali 1,2,a, Pamela Robles ${ }^{1, b}$, Mónica Mendoza $^{2,3, c}$, Celeste Torres ${ }^{2,3, c}$
}

\begin{abstract}
RESUMEN
El arsénico es un elemento que se encuentra ampliamente distribuido en todo el medio ambiente. Sus compuestos se hallan principalmente en estado de oxidación pentavalente y trivalente; y en formas inorgánicas y orgánicas. Las especies arsenicales varían en su grado de toxicidad, siendo los compuestos inorgánicos más tóxicos que los orgánicos, y los compuestos trivalentes más tóxicos que los pentavalentes. Habría interconversión entre las especies menos tóxicas a otras más toxicas y los métodos de cocción y de procesamiento podrían afectarla. El arsénico es un agente carcinogénico y ocasiona múltiples efectos negativos sobre la salud humana a corto y largo plazo. La exposición humana no ocupacional al arsénico se da principalmente por agua y alimentos. La normativa es variable para cada país, y se basa en los estándares de la OMS, Codex Alimentarius y la Unión Europea. Muchos estudios se enfocan en determinar el contenido total de arsénico mas no identifican las especies arsenicales en alimentos. A nivel global, pescado y mariscos, pollo, carnes, arroz y algas marinas tienen niveles elevados de arsénico. En Perú, hay escasos estudios sobre contenido de arsénico total y especies arsenicales en alimentos a pesar que tenemos zonas con altos niveles de contaminación ambiental. El objetivo de esta revisión es discutir la exposición al arsénico a través de la ingesta de alimentos y agua, la normativa relacionada, toxicidad, consecuencias en la salud humana y principales alimentos que contribuyen a su ingesta.
\end{abstract}

Palabras clave: Arsénico; Alimentos; Exposición; Toxicidad; Cáncer (Fuente: DeCS BIREME).

ARSENIC INTAKE: IMPACT IN HUMAN NUTRITION AND HEALTH

\begin{abstract}
Arsenic is an element that is widely distributed throughout the environment. Its compounds are mainly in the state of pentavalent and trivalent oxidation; and in inorganic and organic forms. Arsenical species vary in their degree of toxicity, with inorganic compounds being more toxic than organic and trivalent compounds more toxic than pentavalent compounds. There would be interconversion between the less toxic species and other more toxic species and the cooking and processing methods could affect it. Arsenic is a carcinogenic agent and causes multiple negative effects on human health in the short and long term. Non-occupational human exposure to arsenic occurs mainly through water and food. The regulation is variable for each country and is based on WHO standards, the Codex Alimentarius, and the European Union. Many studies focus on determining the total arsenic content but do not identify arsenical species in foods. Globally, fish and seafood, chicken, meat, rice, and seaweed have high levels of arsenic. In Peru, there are few studies on total arsenic content and arsenical species in food despite the fact that we have areas with high levels of environmental contamination. The objective of this review is to discuss exposure to arsenic through food and water intake, related regulations, toxicity, consequences on human health and main foods that contribute to its intake.
\end{abstract}

Keywords: Arsenic; Foods; Exposure; Toxicity; Cancer (Source: DeCS BIREME)

\section{INTRODUCCIÓN}

El arsénico es un elemento natural de la corteza terrestre, distribuido en todo el medio ambiente y presente en el aire, el agua y la tierra; siendo también causal de contaminación por actividades antropogénicas ${ }^{(1,2)}$.
El arsénico existe en cuatro estados de oxidación: $-3,0,+3 y+5^{(3,4)}$. La mayoría de los compuestos arsenicales en organismos y en alimentos están en el estado de oxidación pentavalente ${ }^{(5)}$.

Los compuestos arsenicales en estados de oxidación trivalente y pentavalente son los de mayor interés en

\footnotetext{
Facultad de Ciencias de la Salud, Universidad Peruana de Ciencias Aplicadas. Lima, Perú.

Facultad de Ciencias de la Salud, Universidad Científica del Sur. Lima Perú.

División de Ciencias Biológicas y de la Salud, Universidad Autónoma Metropolitana, Unidad Xochimilco. Ciudad de México, México

${ }^{\mathrm{a}}$ Ingeniero en Alimentos, Maestro en ciencias; ${ }^{\mathrm{b}}$ nutricionista, Magister en gestión y docencia en alimentación y nutrición; ${ }^{\mathrm{c}}$ nutricionista.

Recibido: 08/08/2017 Aprobado: 17/01/2018 En línea: 05/04/2018
}

Citar como: Medina-Pizzali M, Robles P, Mendoza M, Torres C. Ingesta de arsénico: el impacto en la alimentación y la salud humana. Rev Peru Med Exp Salud Publica. 2018;35(1): 93-102. doi: 10.17843/rpmesp.2018.351.3604. 
estudios ambientales ${ }^{(3)}$ y los podemos clasificar en tres grupos, compuestos arsenicales inorgánicos, compuestos arsenicales orgánicos y la arsina ${ }^{(6)}$. La exposición a la arsina no se relaciona con alimentos ${ }^{(7)}$.

En agua potable, el arsénico se halla en forma inorgánica como arsenato $\left(\mathrm{As}^{+5}\right)$ y arsenito $\left(\mathrm{As}^{+3}\right)^{(8)}$. El arroz contiene principalmente arsénico inorgánico $\left(A s_{\text {in }}\right){ }^{(9,10)}$, además de ácido monometilarsónico $\left(\mathrm{MMA}^{+5}\right)$, ácido dimetilarsínico $\left(\mathrm{DMA}^{+5}\right)$, arsénico tri-glutation (ATG), mono metilarsonico di-glutatión (MADG) y dimetil arso- glutatión (DMAG) (8). Las algas marinas son ricas en arsenoazúcares ${ }^{(8,10)}$ y las setas contienen una variedad de organoarsenicales ${ }^{(10)}$. En algunos peces y crustáceos, la arsenobetaína es la principal especie arsenical hallada ${ }^{(8,11)}$, mientras que en general en los peces los arsenolípidos representan alrededor del 10-

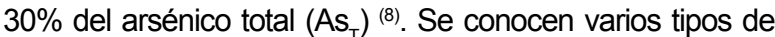
arsenolípidos, entre ellos arsenohidrocarburos, arseno ácidos grasos y arsenofosfolípidos ${ }^{(12)}$. Los peces marinos también contienen $\mathrm{As}_{\text {in }}$ representando alrededor del $10 \% \operatorname{del~} \mathrm{As}_{\mathrm{T}}{ }^{(13)}$. El principal compuesto arsenical hallado en aves de corral es el ácido 3-nitro-4 hidroxifenilarsónico (roxarsona), usado en la producción avícola convencional ${ }^{(14)}$, aquella realizada en forma industrial e intensiva ${ }^{(15)}$. La Tabla 1 muestra los principales compuestos arsenicales relacionados con alimentos.

Para esta revisión narrativa, se buscaron publicaciones en inglés y español con la palabra arsénico en Scopus, Google Scholary Scieloentre los años 2009 hastael2017, aunque por relevancia se incluyeron algunas publicaciones anteriores, restringiendo la búsqueda mediante la combinación de las otras palabras clave con la palabra arsénico. El objeto de esta revisión es discutir la exposición al arsénico a través de la ingesta de alimentos y agua, la normativa relacionada, toxicidad, consecuencias en la salud humana y principales alimentos que contribuyen a su ingesta.

Tabla 1. Principales compuestos arsenicales relacionados con alimentos ${ }^{1}$

\begin{tabular}{|c|c|}
\hline Compuesto arsenical & Se encuentra en \\
\hline Arsenito $\left(\mathrm{As}^{+3}\right)$ y Arsenato $\left(\mathrm{As}^{+5}\right)^{2}$ & $\begin{array}{l}\text { Algas marinas } \\
\text { Setas comestibles } \\
\text { Vegetales } \\
\text { Agua potable } \\
\text { Arroz } \\
\text { Trigo y productos a base de este } \\
\text { Animales de origen marino (en especial bivalvos) } \\
\text { Leche y carne y productos a base estos }\end{array}$ \\
\hline Ácido monometilarsenoso $\left(\mathrm{MMA}^{+3}\right)$ & Setas comestibles \\
\hline Ácido dimetilarsinoso $\left(\mathrm{DMA}^{+3}\right)$ & $\begin{array}{l}\text { Podría encontrarse en diversos organismos por ser un metabolito } \\
\text { inestable de } \mathrm{As}_{\text {in }}\end{array}$ \\
\hline Ácido monometilarsónico $\left(\mathrm{MMA}^{+5}\right)$ & $\begin{array}{l}\text { Arroz } \\
\text { Setas comestibles } \\
\text { Carne de animales }^{3}\end{array}$ \\
\hline Ácido dimetilarsínico $\left(\mathrm{DMA}^{+5}\right)$ & $\begin{array}{l}\text { Arroz } \\
\text { Setas comestibles } \\
\text { Carne de animales }^{3}\end{array}$ \\
\hline Arsénico tri-glutation (ATG) & Arroz \\
\hline Óxido de trimetilarsina (TMAO) & Setas comestibles \\
\hline Ion Tetrametilarsonio $\left(\mathrm{TMA}^{+}\right)$ & $\begin{array}{l}\text { Musculo de animales de origen marino } \\
\text { Setas comestibles }\end{array}$ \\
\hline Arsenobetaína (AB) & $\begin{array}{l}\text { Musculo de animales de origen marino } \\
\text { Setas comestibles }\end{array}$ \\
\hline Arsenocolina (AC) & $\begin{array}{l}\text { Setas comestibles } \\
\text { Pescados }\end{array}$ \\
\hline Arsenoazúcares & $\begin{array}{l}\text { Pescados } \\
\text { Algas marinas }\end{array}$ \\
\hline Arsenolípidos & Pescados grasos y sus aceites \\
\hline Ácido 3 -nitro-4 hidroxifenilarsónico (roxarsona) & Carne de aves de corral, en especial pollos \\
\hline Ácido 4-nitrofenilarsonico (nitrasona) & Carne de aves de corral, es especial pavos \\
\hline
\end{tabular}

${ }^{1}$ Muchos estudios sólo evalúan el contenido de arsénico total, sin indagar la especie arsenical.

2 Muchos estudios no los cuantifican individualmente sino como la suma de ambos, $\mathrm{As}_{\text {in }}$

${ }^{3}$ No siempre se especifica la valencia de DMA y MMA, pero usualmente en alimentos se cuantifican en forma de MMA $^{+5}$ y $\mathrm{DMA}^{+5}$ 


\section{EXPOSICIÓN HUMANA AL ARSÉNICO}

La exposición humana al arsénico puede ocurrir mediante tres vías principales: por inhalación de aire, por ingestión de alimentos y agua, y por absorción dérmica ${ }^{(16)}$.

La exposición al arsénico deriva de varias fuentes como el uso y manufactura de pesticidas y herbicidas, minería y metalurgia, fundición y refinación de metales, uso de combustibles fósiles, uso de medicamentos y remedios, contacto con maderas prensadas y tratadas con preservantes arsenicales, ingesta de agua contaminada y alimentos ${ }^{(2,3,11)}$. La principal forma de exposición no ocupacional al arsénico es a través de alimentos y agua ${ }^{(16,17)}$.

Existen reportes sobre casos de exposición crónica con arsénico por agua de consumo humano, como la pandemia de Bangladesh y Bengala Occidental (4,18), así como en Argentina, Chile, China, India, México, Taiwán, Tailandia y los EUA ${ }^{(18)}$.

En Latinoamérica, se estima que por lo menos 4,5 millones de personas beben en forma permanente agua con niveles de arsénico que ponen en riesgo su salud. La cantidad de arsénico en el agua, especialmente en el agua subterránea, llega en algunos casos a superar la concentración de $1000 \mu \mathrm{g} / \mathrm{L}$. En Perú, su presencia en las fuentes de agua para consumo humano se puede deber a factores naturales de origen geológico, a la explotación minera y a la refinación de metales por fundición ${ }^{(19)}$. Se han reportado niveles de arsénico en agua de consumo humano por encima del actual límite máximo permisible recomendado por la OMS en agua potable de $10 \mu \mathrm{g} / \mathrm{L}$, y en la mayoría de los casos los niveles eran superiores a $50 \mu \mathrm{g} / \mathrm{L}$, (límite anterior recomendado). Por ejemplo, en el año 2002 se encontraron niveles de arsénico en el río Rímac, de hasta $780 \mu \mathrm{g} / \mathrm{L}{ }^{(20)}$. Esto tiene mucho impacto considerando que este río abastece de agua a la ciudad de Lima, donde se concentra la mayor población urbana del país. En la zona sur, se determinó que el agua de los ríos Callazas y Salado, tiene niveles tan altos como 640 y $1680 \mu \mathrm{g} / \mathrm{L}$, respectivamente, también se detectó arsénico en aguas subterráneas en zonas de Puno, Tacna y Moquegua ${ }^{(19)}$.

Se han hecho esfuerzos desde diversos sectores para desarrollar técnicas para remover el arsénico del agua para consumo humano en Latinoamérica, usando tecnologías de bajo costo teniendo en cuenta a las poblaciones vulnerables de riesgo y de bajos recursos económicos. Estas tecnologías varían en eficiencia y la misma es afectada por diversos factores. Por otro lado, habría que considerar el manejo correcto de los residuos tóxicos ${ }^{(21-24)}$. Se estima que en Latinoamérica los alimentos contribuyen hasta con $50 \%$ de la ingesta de $\mathrm{As}_{\mathrm{T}}{ }^{(19)}$. El monitoreo de productos alimenticios indica que el arsénico está presente en todos los alimentos ${ }^{(10,16)}$. Las concentraciones de $\mathrm{As}_{\mathrm{T}}$ en los alimentos procedentes de diversos países varían mucho dependiendo del tipo de alimentos, condiciones (tipo de suelo, el agua, la actividad geoquímica, el uso de pesticidas arsenicales) y técnicas de procesamiento, pero comúnmente, las mayores concentraciones de $\mathrm{As}_{\mathrm{T}}$ se encuentran en pescados y mariscos, seguidos de carnes y granos; las frutas, verduras y lácteos tienden a tener concentraciones totales menores ${ }^{(16)}$. Sin embargo, el arroz y las algas marinas presentan las más altas concentraciones promedio a nivel mundial ${ }^{(25)}$.

En Bolivia, Brasil, Chile, Ecuador, El Salvador, Honduras, México, Nicaragua y Perú se han detectado niveles elevados no solo en pescado, sino también en leche de vaca, granos y hortalizas, incluidas la papa, cebolla, remolacha, calabaza, rábano, col y frijoles ${ }^{(19)}$. La cerveza y el vino también contribuyen a la ingesta de arsénico en la dieta ${ }^{(6)}$.

En los Estados Unidos se estima que la ingesta de arsénico en alimentos está en un rango de 1 a $20 \mu \mathrm{g} \mathrm{As}$ in /día y se han identificado varios alimentos que suelen estar contaminados, entre ellos arroz, algunos jugos y pollo ${ }^{(11)}$. En Canadá la mayor concentración de $\mathrm{As}_{\mathrm{T}}$ se encontró en pescados, seguido de carnes y pollo ${ }^{(26)}$. Estudios en Chile y Brasil hallaron niveles altos de arsénico en diferentes especies de peces excediendo los valores recomendados por la FAO/ OMS y las normas de estos países en esa época ${ }^{(19)}$.

\section{NORMATIVA RELACIONADA}

La normativa relacionada con niveles de arsénico en agua de uso doméstico y alimentos es compleja, pudiendo variar en cada país y con el tiempo ${ }^{(27)}$.

En el año 1993, la Organización Mundial de la Salud redujo su criterio recomendado para agua potable de 50 a $10 \mu \mathrm{g}$ de arsénico/L ${ }^{(27-29)}$. Esto se refleja en los valores establecidos por el Codex Alimentarius para grasas y aceites con 0,1 $\mathrm{mg} / \mathrm{kg}$, grasas para untar y mezclas para untar con $0,1 \mathrm{mg} /$ $\mathrm{kg}$, aguas minerales naturales con $10 \mu \mathrm{g} / \mathrm{L}$, arroz pulido con $0,2 \mathrm{mg} \mathrm{As}$ in $/ \mathrm{kg}$ y sal de mesa con $0,5 \mathrm{mg} / \mathrm{kg}^{(30)}$. Aunque la OMS y el Codex Alimentarius no son entes reguladores con el poder de establecer normas y hacerlas cumplir, su criterio recomendado respecto al arsénico ha sido incorporado a la normativa de diversos países, como el Perú (28).

En los Estados Unidos, para carne y huevos de pollo y de pavo, la FDA limita la cantidad de $A s_{T}$ a $0,5 \mathrm{mg} / \mathrm{kg}$ en los tejidos musculares crudos, $2 \mathrm{mg} / \mathrm{kg}$ en subproductos (órganos) comestibles crudos ${ }^{(31)}$, y $0,5 \mathrm{mg} / \mathrm{kg}$ en los huevos; estableciendo además que los pollos no deben ser alimentados con suplementos de arsénico dentro de los 
cinco días antes del sacrificio ${ }^{(27)}$. En cerdos, los límites de arsénico son $2 \mathrm{mg} / \mathrm{kg}$ en el hígado y los riñones sin cocer, y $0,5 \mathrm{mg} / \mathrm{kg}$ en los tejidos musculares crudos y productos derivados distintos de hígado y los riñones ${ }^{(27)}$. La FDA no ha establecido nuevos estándares para otros alimentos que han reportado contaminación por arsénico como arroz y jugos y los valores límites para aves de corral no han sido

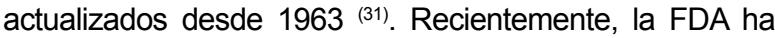
establecido límites no obligatorios para productos a base

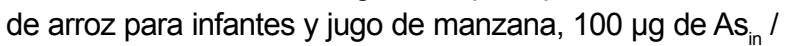
$\mathrm{kg}$ y $10 \mu \mathrm{g}$ de $A s_{\text {in }} / \mathrm{kg}$, respectivamente ${ }^{(32,33)}$.

En el 2015, la Unión Europea fijó los valores para los contenidos máximos de $\mathrm{As}_{\text {in }}$ en arroz y sus productos derivados, siendo de $0,20 \mathrm{mg} / \mathrm{kg}$ en arroz pulido y $0,25 \mathrm{mg} /$ kg para arroz sancochado ${ }^{(34)}$.

\section{ABSORCIÓN Y TOXICIDAD DEL ARSÉNICO}

La matriz de los alimentos afecta a la absorción de compuestos arsenicales en el tracto gastrointestinal. Los compuestos arsenicales hidrosolubles se absorben con mayor facilidad que los liposolubles. Estudios en roedores indican una casi completa absorción de arsénico inorgánico presente en el agua potable (95\%). La tasa de absorción del $\mathrm{As}_{\text {in }}$ en arroz fue del $89 \%$ en un modelo basado en cerdos ${ }^{(5)}$. La bioaccesibilidad del $\mathrm{As}_{\text {in }}$ y el $\mathrm{DMA}^{+5}$ podría reducirse por la presencia de ciertos componentes de la matriz alimenticia en cuestión ${ }^{(35)}$.

El arsénico puede causar múltiples alteraciones en un número de procesos moleculares y celulares, y estas tienen un papel en la manifestación de su toxicidad ${ }^{(2,4,11,17,36-40)}$, los cuales se muestran en la Tabla 2.

La toxicidad del arsénico depende de su forma y su estado de oxidación, además de otros factores, tales como dosis, duración y frecuencia de exposición, especie biológica en estudio; edad; sexo; sensibilidad individual; genética; y factores nutricionales ${ }^{(17)}$. En el cuerpo humano, se dan una serie de reducciones sucesivas y reacciones oxidativas, así como reacciones de metilación, que transforman el $\mathrm{As}_{\text {in }}$ para producir diferentes metabolitos ${ }^{(2,}$. La toxicidad de cada metabolito es un reflejo de su estado de valencia, que determina su reactividad, la vida media y la distribución en el cuerpo. Como resultado, cada especie arsenical puede tener diferentes efectos, dando lugar a grandes diferencias en la toxicidad ${ }^{(2-16)}$.

El arsénico se encuentra en alimentos como una mezcla de compuestos inorgánicos e orgánicos, considerándose estos últimos como los menos tóxicos $(2,4,16,17)$. Las formas trivalentes son más tóxicas que las pentavalentes $(3,16,17,42)$ $\mathrm{y}$, las formas pentavalentes $\mathrm{y}$ trivalentes se pueden interconvertir fácilmente ${ }^{(43)}$. A pesar de que los compuestos arsenicales inorgánicos son más tóxicos que los orgánicos hay evidencia de que el $\mathrm{MMA}^{+5}$ y el $\mathrm{DMA}^{+5}$, productos derivados del metabolismo de desintoxicación del arsénico en humanos, son promotores de cáncer ${ }^{(6)}$. A su vez, se ha reportado que el $\mathrm{MMA}^{+3}$ es más citotóxico que el arsénico inorgánico trivalente ${ }^{(41)}$. Dentro de los compuestos arsenicales orgánicos, las formas metiladas son más tóxicas que la arsenobetaína y arsenocolina, siendo estos últimos considerados prácticamente inocuos ${ }^{(3,6)}$ aunque la arsenobetaína podría transformarse en el ion $\left(\mathrm{TMA}^{+}\right)$a altas temperaturas de cocción ${ }^{(44)}$.

Los arsenoazúcares son menos tóxicos que las especies arsenicales metiladas pero existe evidencia de su potencial toxicidad ${ }^{(3)}$. Se demostró que los arsenoazúcares trivalentes son más tóxicos que los pentavalentes y que una especie de arsenoazúcar trivalente presenta tanta capacidad para dañar el ADN como la mostrada por el $\mathrm{DMA}^{+3}{ }^{(43)}$. Por otro lado, los arsenolípidos han demostrado ser potentes agentes citotóxicos similares en su acción al $\mathrm{As}^{+3(5)}$.

\section{EFECTOS EN LA SALUD HUMANA DE LA EXPOSICIÓN AL ARSÉNICO}

Existen pocos estudios epidemiológicos sobre los efectos en la salud de los niveles de arsénico en alimentos, ya que mayormente la data disponible proviene de estudios realizados en agua y en poblaciones expuestas a altas dosis ${ }^{(45)}$.

Los efectos de la exposición al arsénico van desde la letalidad aguda a los efectos crónicos. Existen múltiples consecuencias, viéndose afectados varios sistemas y órganos diferentes, incluyendo la piel y las vías respiratorias, sistema cardiovascular, inmunológico, genitourinario, reproductivo, digestivo, nervioso ${ }^{(16)}$, así como el sistema eritropoyético, endocrino, hepático y renal ${ }^{(37)}$.

\section{EFECTOS A CORTO PLAZO}

La ingestión de grandes dosis de arsénico por lo general resulta en síntomas en un plazo de 30 a 60 minutos. La intoxicación aguda habitualmente comienza con un sabor metálico, sensación quemante en los labios y disfagia $(7,16)$. Pueden sobrevenir diversos síntomas gastrointestinales, entre ellos, dolor abdominal y cólicos, diarrea profusa, naúsea y vómitos violentos ${ }^{(7)}$. Los vómitos eventualmente pueden conducir a la hematemesis ${ }^{(16)}$. Los síntomas gastrointestinales a menudo conllevan a la deshidratación y el desequilibrio electrolítico, y pueden conducir a la hipotensión y la hipoxia ${ }^{(16)}$. Después de los síntomas iniciales, pueden ocurrir fallos múltiples de órganos ${ }^{(16)}$ especialmente en los sistemas cardiovascular, renal y hepático, causando la muerte ${ }^{(7)}$. 
Tabla 2. Principales procesos moleculares y celulares involucrados con la toxicidad al arsénico

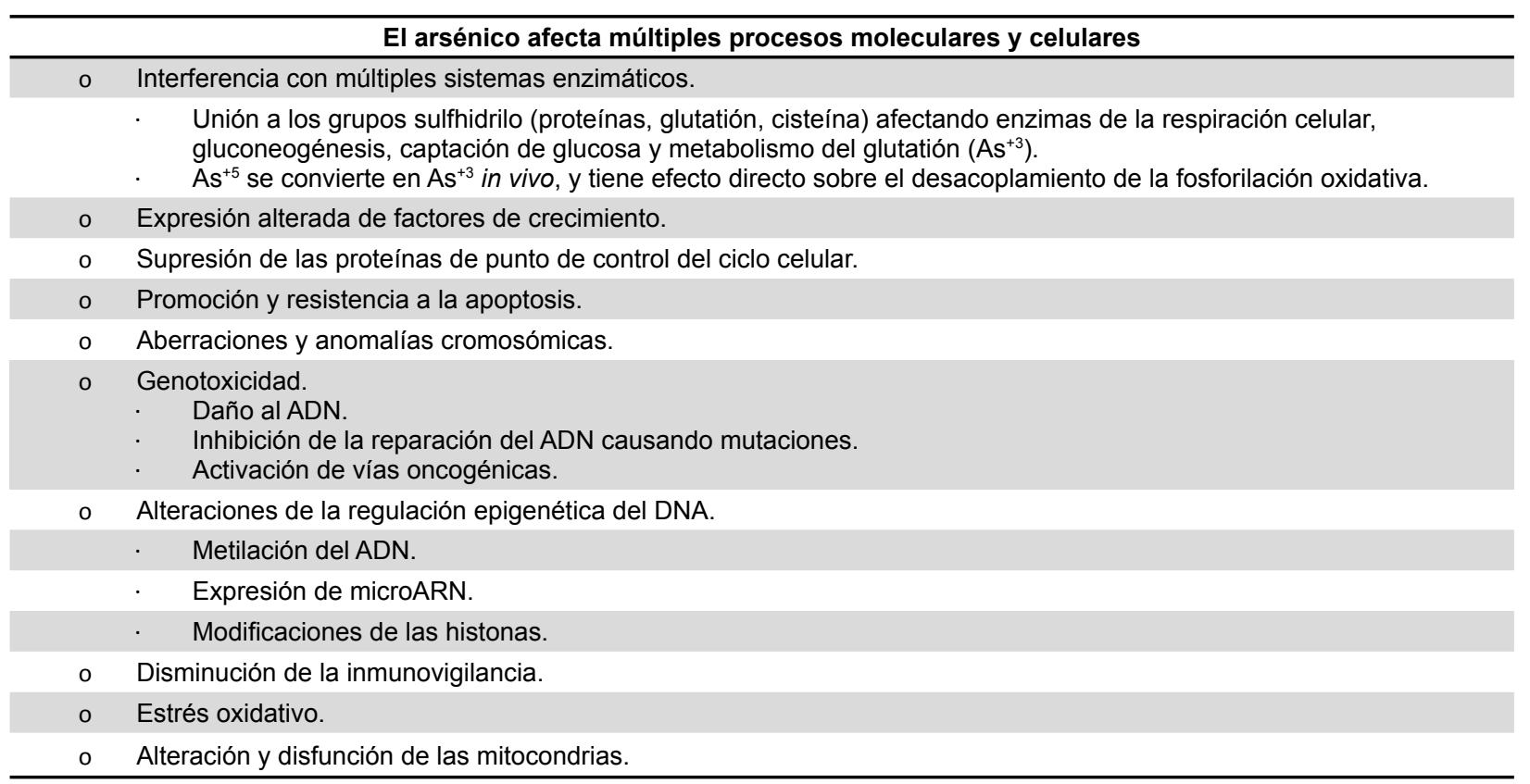

\section{EFECTOS CRÓNICOS}

Una infinidad de efectos sobre la salud están relacionados con la exposición crónica al arsénico afectando casi todos los órganos y principales sistemas del cuerpo ${ }^{(2,4,7,17,19,36,37,46-50)}$, los cuales se pueden visualizar en forma esquemática en la Figura 1.
En Argentina, se conoce como hidroarsenicismo crónico regional endémico (HACRE) a la patología provocada por la ingesta crónica de arsénico en el agua y los alimentos, siendo un problema de salud pública de larga data en ese país. Provoca afecciones de piel, cáncer de piel, pulmón, vejiga, riñón, entre otros, alteraciones en el desarrollo, afecciones cardiovasculares, neurotoxicidad y diabetes ${ }^{(51)}$. Por otro lado,

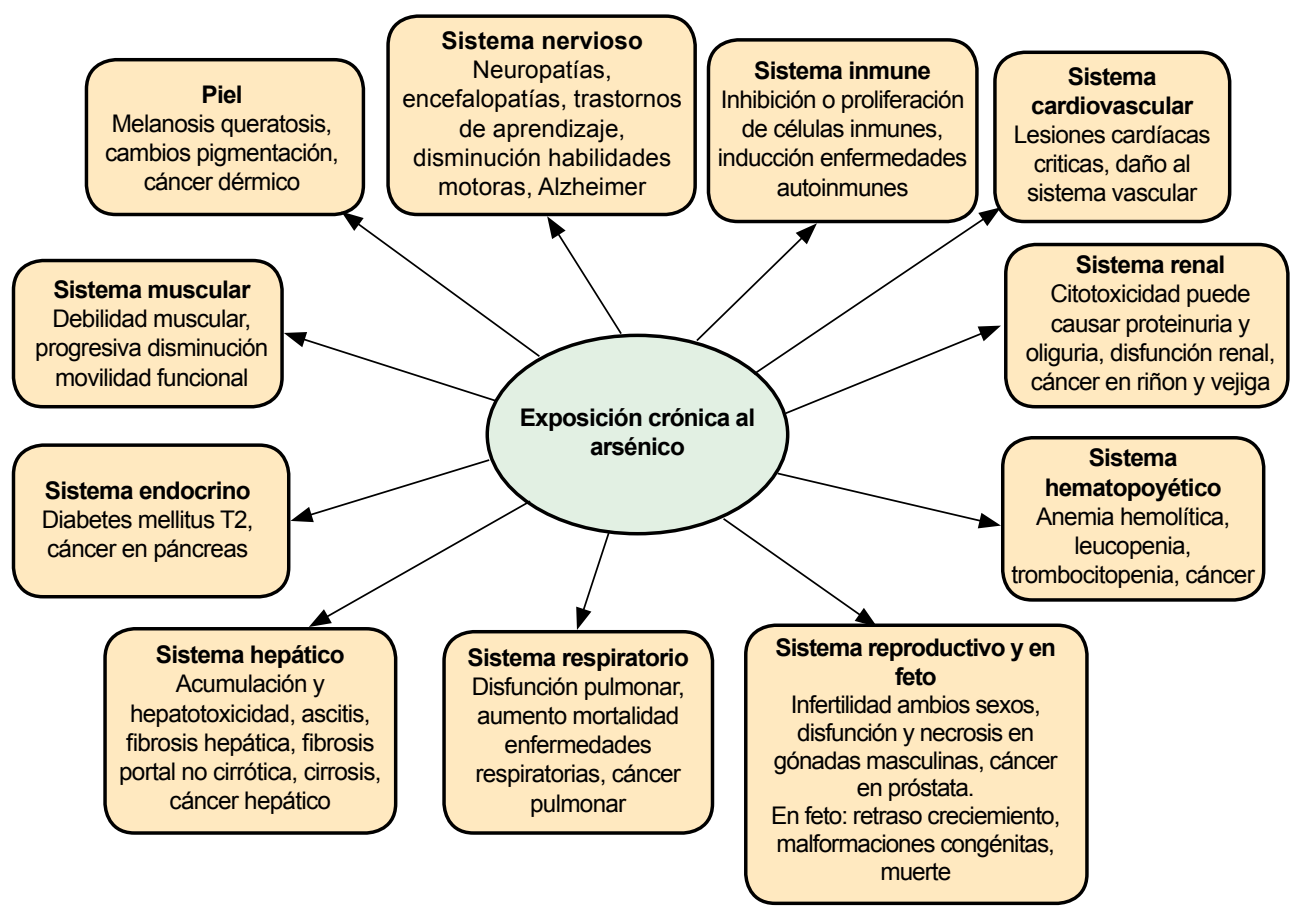

Figura 1. Diagrama esquemático de los efectos de la exposición crónica al arsénico 
la Enfermedad del Pie Negro está asociada con la ingesta crónica de arsénico a través del agua potable en Taiwán desde 1920 , ocasionando gangrena en los pies ${ }^{(3)}$.

\section{INCIDENCIA DE CÁNCER}

El arsénico fue reconocido como sustancia cancerígena en 1980 y registrado como tal en la Agencia Internacional para la Investigación sobre el Cáncer (IARC) ${ }^{(17,37)}$

La actividad carcinogénica del arsénico se relaciona con su proceso de biotransformación, la cual ocurre a través de una serie de reacciones reducción, oxidación y metilación ${ }^{(41)}$, teniendo efectos a niveles genéticos y epigenéticos ${ }^{(39)}$.

Los resultados de estudios epidemiológicos y de animales han demostrado que los compuestos inorgánicos de arsénico pueden ser categorizados como claramente carcinógenos (grupo 1) o carcinógenos potenciales (grupo 2B), mientras que otros como la arsenobetaína y otros órgano-arsenicales no han sido clasificados como carcinógenos (grupo 3) (17). Hasta la fecha, la IARC ha confirmado la asociación de la exposición al arsénico con el cáncer de la piel, los pulmones y vejiga, y se ha demostrado su capacidad de inducir tumores cancerosos en el hígado, riñón y próstata ${ }^{(17,37,46)}$. Otros estudios relacionan la exposición al arsénico con el cáncer en páncreas, y en el sistema eritropoyético ${ }^{(49)}$.

El cáncer de pulmón es el cáncer más conocido asociado con la exposición al arsénico. Se ha estudiado principalmente la relación entre el cáncer de pulmón y la exposición a altos niveles de arsénico, la relación entre este cáncer con la exposición a bajos niveles son insuficientes o no concluyentes ${ }^{(17,37,45)}$. Podría existir sinergia entre la ingesta de arsénico y el tabaquismo ${ }^{(52,53)}$.

La exposición al $\mathrm{As}^{+3}$ se asocia con cánceres de piel inducidos por radiación UV ${ }^{(13)}$. Los cánceres de piel asociados con el arsénico son generalmente carcinomas de células basales o de células escamosas causadas por queratinización ${ }^{(7,13,46)}$.

La asociación entre el cáncer de vejiga y el arsénico en agua potable ha sido verificada a altas concentraciones de arsénico ${ }^{(17) ;}$ sin embargo, la evidencia sugiere que existe un mayor riesgo a niveles tan bajos como $10 \mu \mathrm{g} / \mathrm{L}$ en agua potable ${ }^{(54)}$. A altos niveles de exposición al arsénico, el tabaquismo interactúa sinérgicamente, aumentando el riesgo de cáncer de vejiga ${ }^{(17,19)}$.

Amplia evidencia demuestra que el hígado es un órgano diana de carcinogenicidad para el arsénico ${ }^{(47)}$. El desarrollo de cáncer de hígado se asocia con exposición a altos niveles de arsénico en agua potable ${ }^{(55)}$. Estudio de cohortes retrospectivos indican una mayor incidencia y mortalidad de cáncer de hígado en sujetos expuestos al arsénico en etapas tempranas de su vida ${ }^{(17}$,

\section{INGESTA DE ARSÉNICO A TRAVÉS DE ALIMENTOS DE ORIGEN ANIMAL}

Los pescados y mariscos son el principal contribuyente de arsénico en la dieta ${ }^{(12)}$. En estos alimentos, el arsénico se halla principalmente en forma orgánica, pero este se reporta como $\mathrm{As}_{\mathrm{T}}$. Los niveles de organoarsenicales están entre 1-100 mg As/kg de peso húmedo. La arsenobetaína es el compuesto arsenical más frecuente en los organismos marinos ${ }^{(5)}$. Los alimentos de origen marino contienen bajos niveles de $A s_{\text {in }}$, generalmente $<0,2 \mathrm{mgAs}_{\text {in }} / \mathrm{kg}$ de peso seco. Sin embargo, los bivalvos como los choros (Mytilus edulis), tienen concentraciones relativamente altas, oscilando entre 0,001 a $4,5 \mathrm{mg} \mathrm{As}_{\text {in }} / \mathrm{kg}^{(5)}$.

Los arsenolípidos se encuentran mayormente en peces grasos y en sus aceites ${ }^{(12)}$, como el aceite de hígado de bacalao, capelán y atún ${ }^{(5)}$. Los arsenohidrocarburos parecen predominar en especies de peces con mayor contenido total de arsenolípidos (rombo y sardina), mientras que los arseno ácidos grasos parece predominar en aquellas con menor contenido total de arsenolípidos (caballa y salmonetes) ${ }^{(5)}$.

Un estudio realizado en España ${ }^{(13)}$ mostró que el consumo de pescado blanco en adultos y niños, y de pescado azul en el caso de los adultos, presentaría un riesgo carcinogénico moderado para los consumidores españoles, sobre todo en relación con sus concentraciones de arsénico. Se estimó que los productos marinos en adultos aportan 1,96 $\mu \mathrm{g} \mathrm{As} / \mathrm{kg} / \mathrm{día}$, lo cual representaba el $94 \%$ de la ingesta diaria tolerable de la FAO/OMS $(2,1 \mu \mathrm{g} / \mathrm{kg} / \mathrm{día}){ }^{(13)}$. Cabe agregar que este valor fue retirado por ser considerado muy elevado ${ }^{(1)}$.

En Argentina, se determinaron las concentraciones de $\mathrm{As}_{\mathrm{T}}$ en varios tejidos de bovino destinados a consumo humano, los cuales fueron riñón, hígado, músculo esquelético y glándulas mamarias. Los niveles de arsénico en riñón e hígado fueron superiores a los hallados en músculo y glándula mamaria. Las concentraciones halladas en hígado y riñón fueron similares a los valores informados en Canadá, Países Bajos, Australia y Galicia en estudios previos ${ }^{(56)}$.

Esimportante resaltarque, en ambosestudios mencionados, solo se determinó $\mathrm{As}_{\mathrm{T}}$. Esto es una limitación ya que se sabe que es preciso determinar las especies arsenicales a fin de poder evaluar su toxicidad correctamente.

El arsénico se encuentra en pollos, pavos y cerdos, debido al uso de drogas organoarsenicales añadidas a los piensos. La roxarsona, la carbasona y el ácido p-arsanílico, fueron retirados del mercado en los EE.UU en 2013, pero la nitrasona o ácido 4-nitrofenilarsonico, se utilizó en la producción de pavos hasta el $2015^{(14)}$. En Perú, la roxarsona tiene registro vigente como insumo agropecuario ${ }^{(58)}$. 
La decisión de discontinuar el uso de roxarsona y compuestos similares se basó en un estudio que caracterizó las especies arsenicales encontradas en pollo, estimando los riesgos asociados al cáncer de pulmón y de vejiga. La carne cruda de pollo convencional contenía roxarsona y $\mathrm{As}_{\text {in }}$ derivado del mismo, estando este último en mayores concentraciones que en otros tipos de carne de pollo. Se reportó que la cocción aumenta la conversión de roxarsona en $\mathrm{As}_{\text {in }}$ en el músculo de pollo ${ }^{(31)}$. Esto podría ser un punto de partida para estudios en nuestro país que comparen especies arsenicales en alimentos antes y después de la cocción, especialmente pollo, dado que su consumo es masivo en Perú (59).

\section{INGESTA DE ARSÉNICO A TRAVÉS DE ALIMENTOS DE ORIGEN VEGETAL}

Las vegetales son de interés desde el punto de vista toxicológico debido a que crecen en suelos contaminados. El arroz presenta la mayor concentración de especies de $\mathrm{As}_{\text {in }}$ en comparación con otros productos vegetales ${ }^{(42,60)} \mathrm{y}$ es frecuentemente la principal ruta de exposición humana cuando las concentraciones de arsénico en agua potable son menores a $50 \mu \mathrm{g} / \mathrm{L}^{(38)}$. El arroz integral contiene mayores niveles de arsénico que el arroz pulido ${ }^{(60)}$. El arroz se usa en muchos países en la elaboración de alimentos infantiles siendo una fuente potencial de exposición en niños ${ }^{(2)}$ y en general en toda la población, por ser un alimento básico de la canasta familiar ${ }^{(38)}$. Este cultivo también incorpora metales pesados presentes en el suelo o agua, como cadmio y mercurio. Se estima que el contenido de arsénico en el arroz y alimentos a base de este podría ser de hasta 100 veces mayor que en frutas, mariscos o carne ${ }^{(2)}$ y que el $10-50 \%$ del arroz consumido a nivel mundial tendría una concentración de arsénico mayor a $200 \mu \mathrm{g} / \mathrm{kg}{ }^{(38)}$. Se podría disminuir la concentración de $\mathrm{As}_{\text {in }}$ en arroz, mediante repetidos lavados y cocción en grandes cantidades de agua ${ }^{(25,61,62)}$ y además eliminando el exceso de agua al final ${ }^{(63)}$.

En Argentina, se detectaron concentraciones elevadas de $\mathrm{As}_{\mathrm{T}}$ en tres provincias productoras de arroz, en un rango de 0,451 a 0,690 $\mu \mathrm{g} / \mathrm{g}$ (peso seco) ${ }^{(51)}$. En Perú, el arroz es un alimento básico, con un consumo de $48 \mathrm{Kg} /$ persona/ año ${ }^{(64)}$ y sería necesario saber su concentración de $\mathrm{As}_{\mathrm{T}} \mathrm{y}$ determinar las especies arsenicales .

En Chile, se determinó el contenido de $\mathrm{As}_{\mathrm{T}}$, las especies arsenicales y su bioaccesibilidad en zanahoria, remolacha y quinua. Se encontró que el $\mathrm{As}^{+3}$ y $\mathrm{As}^{+5}$ eran las únicas especies presentes en las zanahorias y la quinua. La quinua acumuló la mayor cantidad de $\mathrm{As}_{\text {in }}$, pero su bioaccesibilidad era reducida. Por el contrario, para las zanahorias y remolachas la bioaccesibilidad fue de cerca del $100 \%$, pero contenían arsénico $\mathrm{As}^{+5}$ en bajas concentraciones ${ }^{(42)}$.

En algas marinas predominan los arsenoazúcares ${ }^{(12,14,43)}$, pero algunas como, Hizikia fusiforme(hijiki), presentan una concentración extremadamente alta de $\mathrm{As}_{\text {in }}{ }^{(14)}$. La exposición a arsenoazúcares es relativamente alta en Asia debido al consumo de algas, las que contienen niveles de arsénico de hasta $100 \mathrm{mg} / \mathrm{kg}$ de peso seco. Esto podría generar toxicidad crónica dado que los arsenoazúcares se metabolizan a DMA ${ }^{(43)}$. El lavado y remojo del alga disminuiría su contenido de arsénico ${ }^{(25,65)}$. La carragenina al ser un aditivo común en alimentos y un derivado de las algas marinas, podría estar contribuyendo a la exposición al arsénico, aunque se usa en cantidades muy pequeñas ${ }^{(25)}$.

Se ha reportado compuestos arsenicales inorgánicos y orgánicos en setas silvestres y cultivadas, predominando especies inorgánicas, además de $\mathrm{DMA}^{+5}{ }^{(66)}$. Sería importante realizar estudios en Perú respecto a setas usadas en pizzas y ensaladas.

\section{CONCLUSIONES}

La principal forma de exposición no ocupacional al arsénico es a través de alimentos y agua. Pescado y mariscos, pollo, carnes, arroz y algas marinas contienen valores elevados de arsénico, por lo tanto, la determinación del contenido de $\mathrm{As}_{\mathrm{T}}$ como de las especies arsenicales en estos alimentos merece más estudios en nuestro país, donde el pollo y el arroz son muy consumidos.

En el Perú existe contaminación arsenical en fuentes de agua para consumo humano ${ }^{(19)}$. Sería necesario determinar la concentración actual en agua y en alimentos en diferentes zonas y proponer acciones para remediar y prevenir esta contaminación, considerando que la acumulación de arsénico en alimentos dependerá de su concentración en el agua, en el suelo, del uso de pesticidas arsenicales, de las técnicas de procesamiento y cocción, entre otros factores.

La normativa referente al arsénico varía según el país. Para el agua potable, tenemos el estándar de la OMS y el Codex Alimentarius, de $10 \mu \mathrm{g} \mathrm{As}_{\mathrm{T}} / \mathrm{L}$, la cual se aplica en Perú bajo nuestra propia normativa. Los límites para el arroz, en la Unión Europea, están alineados con los del Codex. La FDA estableció límites no obligatorios de $100 \mu \mathrm{g}$ de $\mathrm{As}_{\text {in }} / \mathrm{kg}$ y 10 $\mu \mathrm{g}$ de $\mathrm{As}_{\mathrm{in}} / \mathrm{kg}$, para cereales a base de arroz para infantes y jugo de manzana respectivamente. Asimismo, prohibió el uso de roxarsona y nitrasona para producción de pollos y pavos, lo cual no ha sucedido en Perú.

Se sabe que la exposición crónica al arsénico afecta casi todos los órganos y sistemas, pudiendo causar principalmente cáncer de pulmón, vejiga, de piel, hígado, riñón y próstata. Su absorción es mayor si está en forma hidrosoluble, como en el caso del arroz, pero su bioaccesibilidad podría reducirse por compuestos de la matriz alimenticia.

Los estudios de determinación de especies arsenicales en alimentos son necesarios ya que su toxicidad varía según la 
especie, entre otros factores. Generalmente, su toxicidad es mayor en $\mathrm{As}^{+3}, \mathrm{As}^{+5}$, organoarsenicales metilados trivalentes, organoarsenicales metilados pentavalentes y finalmente menor en otros organoarsenicales como arsenoazúcares, arsenolípidos, y arsenobetaína. Sin embargo, los arsenolípidos y el $\mathrm{MMA}^{+3}$ podrían ser tan o más citotóxicos que el $\mathrm{As}^{+3}$. La interconversión entre las especies menos tóxicas a otras más tóxicas, podría incrementarse debido a métodos de cocción y de procesamiento. Sería deseable tomar medidas en la población para disminuir la exposición, como el remojo y lavados sucesivos de algas y arroz.

Contribución de los autores: MMP ha sido la responsable de la concepción y diseño del artículo, recolección de información bibliográfica, redacción del artículo, revisión crítica del artículo, y obtención de financiamiento. PR ha participado en la concepción del artículo, la recolección de la información bibliográfica y revisión crítica del artículo. Además, MM, y CT han participado en la recolección de la información bibliográfica, y en la revisión crítica del artículo. Todos aprobaron la versión final del artículo.

Fuentes de financiamiento: Cuarto Concurso Anual de Incentivo a la investigación (2016), Universidad Peruana de Ciencias Aplicadas, UPC.

Conflictos de interés: Los autores declaran no tener conflictos de interés.

Agradecimientos: Agradecemos a Ariana Cajero de la Universidad Autónoma Metropolitana, por su aporte a este artículo.

\section{REFERENCIAS BIBLIOGRÁFICAS}

1. FAO/WHO. Seventy-second meeting Rome, 16-25 February 2010. In: Joint FAO/WHO Commmitee on food Additives [Internet]. Rome: FAO; 2010. p. 1-16.[Citado el 06 de junio de 2017] Available from: http://www.who.int/entity/ foodsafety/chem/summary72_rev.pdf

2. Yosim A, Kathryn B, Fry RC. Arsenic, the "King of Poisons," in Food and Water. Am Sci Sci. 2015;103(1):34-41.

3. Hughes MF, Beck BD, Chen Y, Lewis AS, Thomas DJ. Arsenic exposure and toxicology: A historical perspective. Toxicol Sci. 2011;123(2):305-32.

4. Jomova K, Jenisova Z, Feszterova M, Baros S, Liska J, Hudecova D, et al. Arsenic: toxicity, oxidative stress and human disease. J Appl Toxicol. 2011;31(2):95-107.

5. Molin M, Ulven SM, Meltzer HM Alexander J. Arsenic in the human food chain, biotransformation and toxicology - Review focusing on seafood arsenic. J Trace Elem Med Biol. 2015;31:249-59.

6. Cameán, Ana María, and Repetto M. Toxicología alimentaria. Ediciones Díaz de Santos; 2006.705 p. España.

7. Saha JCJ, Dikshit AKA, Bandyopadhyay M, Saha KC. A Review of Arsenic Poisoning and its Effects on Human Health. Crit Rev Environ Sci Technol. 1999;29(3):281-313.

8. Chávez-Capilla T, Beshai M, Maher W, Kelly T, Foster S. Bioaccessibility and degradation of naturally occurring arsenic species from food in the human gastrointestinal tract. Food Chem. 2016;212:189-97.

9. Meharg AA, Raab A. Getting to the bottom of arsenic standards and guidelines. Environ Sci Technol. 2010;44(12):4395-9.

10. JECFA. Evaluation of certain contaminants in food. World Heal Organ Tech Rep Ser. 2011;(959):1-105.

11. Goldman R. Arsenic Exposure and poisoning. UpToDate [Internet]. 2017;123. [Citado el 06 de junio de 2017] Available from: https://www.uptodate.com/ contents/arsenic-exposure-and-poisoning/ print 'source $=$ search_result\&search $=$ arsenic exposure\&selectedTitle $=1 \sim 100$

12. Taylor V, Goodale B, Raab A, Schwerdtle T, Reimer K, Conklin S, et al. Human exposure to organic arsenic species from seafood. Sci Total Environ. 2017;580:26682.

13. Rodriguez-Hernandez A, Camacho M, Henriquez-Hernandez LA, Boada LD, Ruiz-Suarez N, Valeron PF, et al. Assessment of human health hazards associated with the dietary exposure to organic and inorganic contaminants through the consumption of fishery products in Spain. Sci Total Environ. 2016;557-558:808-18.

14. Jackson B, Punshon T. Recent Advances in the Measurement of Arsenic, Cadmium, and Mercury in Rice and Other Foods. Curr Environ Heal Reports. 2016;2(1):15-24.

15. Gerber P, Opio C, Steinfeld H. Poultry production and the environment. A review. FAO;2007.1-27p [Citado el 06 de junio de 2017] Available from: http:// www.fao.org/ag/againfo/home/events/ bangkok2007/docs/part2/2_2.pdf

16. Abernathy, C. and Morgan A. Exposure and Health Effects. Un synthesis report on arsenic in drinking water [Internet].
Geneva, Switzerland: World Health Organization; 2001 [Citado el 06 de junio de 2017]. Available from: http://www. who.int/water_sanitation_health/dwq/ arsenicun3.pdf

17. Hong Y-S, Song K-H, Chung J-Y. Health effects of chronic arsenic exposure. J Prev Med Public Health. 2014; 47(5):245-52.

18. Smith AH, Lingas EO, Rahman M. Contamination of drinking-water by arsenic in Bangladesh: A public health emergency. Bull World Health Organ. 2000;78(9):1093-103

19. McClintock TR, Chen Y, Bundschuh J, OliverJT, NavoniJ, Olmos V, et al. Arsenic exposure in Latin America: biomarkers, risk assessments and related health effects. Sci Total Environ. 2012;429:76-91.

20. Gonzales GF, Zevallos A, GonzalesCastaneda C, Nunez D, Gastanaga C, Cabezas $\mathrm{C}$, et al. Environmental pollution, climate variability and climate change: a review of health impacts on the Peruvian population. Rev Peru Med Exp Salud Publica .2014;31(3):547-56.

21. Rojas-Chaves P, Vargas-Benavides MJ, Araya- A, Valverde-Cerdas J, RomeroEsquivel LG. Estudio de remoción de arsénico en agua potable a nivel domiciliar mediante oxidación solar y coagulaciónfloculación. Tecnol en Marcha. 2015;28(506):55-64.

22. Pellizzari EE, Marinich LG, Flores SA, Giménez CM. Degradación de arsénico por Pseudomonas aeruginosa para bioremediación de agua. Estudio preliminar. Av en Ciencias e Ing. 2015;6(1):1-5.

23. Iliná $\mathrm{A}$, Martínez-Hernández JL, SeguraCeniceros EP, Villareal-Sánchez JA, 
Gregorio-Jaúregui KM. Biosorción de arsénico en materiales derivados de maracuyá. Rev Int Contam Ambient. 2009;25(4):201-6.

24. Chávez Quijada ML, Miglio Toledo M. Remoción de arsénico por oxidación solar en aguas para consumo humano. Soc Quím Perú. 2011;77(4):307-14.

25. Lynch HN, Greenberg GI, Pollock MC, Lewis AS. A comprehensive evaluation of inorganic arsenic in food and considerations for dietary intake analyses. Sci Total Environ. 2014;(496):299-313.

26. Püssa T. Toxicological issues associated with production and processing of meat. Meat Sci. 2013;95(4):844-53.

27. Henke K. Regulation of Arsenic: A Brief Survey and Bibliography. In: Henke K, editor. Arsenic Environmental Chemistry, Health Threats and Waste Treatment [Internet]. John Wiley \& Sons; 2009. p. 54568. Available from: http://onlinelibrary. wiley.com/doi/10.1002/9780470741122. fmatter/pdf

28. OMS. Guías para la calidad del agua potable. Primer Apendice a la Tercera Edicion. Volumen 1. Recomendaciones. Vol. 1. Ginebra: OMS; 2006. p. 398 [Citado el 06 de junio de 2017]. Available from: http://201.147.150.252:8080/xmlui/bitstream/handle/123456789/1262/Investigao_e_evoluo.pdf?sequence $=1$

29. World Health Organization. Guidelines for Drinking Water Quality.First Edition. Vol. 1. Reccomendations [Internet]. Geneva, Switzerland; 1984: Who; p. 148 [Citado el 06 de junio de 2017]. Available from: http://apps.who.int/iris/bitstream/10665/ 252072/1/9241541687-eng.pdf ?ua=1

30. Codex Alimentarius. General Standard for Contaminants and Toxins in Food and Feed. Codex Aliment Int Food Stand [Internet]. 2016;1-59 [Citado el 06 de junio de 2017]. Available from: http://www.fao.org/fao-who-codexalimentarius/sh-proxy/en/? $\operatorname{lnk}=1 \& \mathrm{u}$ $\mathrm{rl}=$ https\%253A\%252F\%252Fworkspace.fao.org\%252Fsites $\% 252$ Fcodex$\% 252$ FStandards\%252FCODEX\%2BSTAN\%2B193-1995\%252FCXS_193s.pdf

31. Nachman KE, Baron PA, Raber G, Francesconi KA, Navas-Acien A, Love DC. Roxarsone, inorganic arsenic, and other arsenic species in chicken: a U.S.Based market basket sample. Env Heal Perspect. 2013;121(7):818-24.
32. Food and Drug Administration (FDA). Draft Guidance for Industry Arsenic in Apple Juice: Action Level Draft Guidance. Washington, U.S. Department of Health and Human Services Food and Drug Administration Center for Food Safety and Applied Nutrition. 2013 [Citado el 06 de junio de 2017]. Available from: https://www.fda.gov/downloads/Food/ GuidanceRegulation/GuidanceDocumentsRegulatoryInformation/ChemicalContaminantsMetalsNaturalToxinsPesticides/UCM360048.pdf.

33. Food and Drug Administration (FDA). U.S. Food and Drug Administration Supporting Document for Action Level for Inorganic Arsenic in Rice Cereals for Infants [Internet]. Washington:FDA; 2016 [Citado el 06 de junio de 2017]. Available from: https://www.fda.gov/downloads/ Food/GuidanceRegulation/GuidanceDocumentsRegulatoryInformation/ UCM493152.pdf

34. Comision Europea. Reglamento (UE) 2015/1006 de la Comisión de 25 de junio de 2015 por el que se modifica el Reglamento (CE) 1881/2006 en cuanto al contenido máximo de arsénico inorgánico en los productos alimenticios. D of la Unión Eur. 2015;(161):14-6.

35. Clemente MJ, Devesa V, Vélez D. Dietary Strategies to Reduce the Bioaccessibility of Arsenic from Food Matrices. J Agric Food Chem. 2016;64(4):923-31.

36. Ambrosio F, Brown E, Stolz D, Ferrari R, Goodpaster B, Deasy B, et al. Arsenic induces sustained impairment of skeletal muscle and muscle progenitor cell ultrastructure and bioenergetics. Free Radic Biol Med. 2014;74:64-73.

37. Abdul KSM, Jayasinghe SS, Chandana EPS, Jayasumana C, Silva PMCS De.EPS, Jayasumana C, Silva PMCS De. Arsenic and human health effects: A Review. Environ Toxicol Pharmacol. 2015;40(3):82846. doi: 10.1016/j.etap.2015.09.016.

38. Banerjee M, Banerjee N, Bhattacharjee P, Mondal D, Lythgoe PR, Martínez M, et al. High arsenic in rice is associated with elevated genotoxic effects in humans. Sci Rep. 2013;3:2195.

39. Hubaux R, Becker-Santos DD, Enfield KS, Lam S, Lam WL, Martinez VD. Arsenic, asbestos and radon: emerging players in lung tumorigenesis. Environ Health. 2012; 11:89. doi: 10.1186/1476-069X11-89.

40. Chanda S, Dasgupta UB, Mazumder DG, Saha J, Gupta B. Human GMDS gene fragment hypermethylation in chronic high level of arsenic exposure with and without arsenic induced cancer. Springerplus. 2013;2(2010):557.

41. Kurzius-Spencer M, Burgess JL, Harris RB, Hartz V, Roberge J, Huang $S$, et al. Contribution of diet to aggregate arsenic exposures-An analysis across populations.J Expo Sci Environ Epidemiol [Internet]. 2014;(24):156-62.

42. Pizarro I, Gómez-Gómez M, León J, Román D, Palacios MA. Bioaccessibility and arsenic speciation in carrots, beets and quinoa from a contaminated area of Chile. Sci Total Environ. 2016;565:557-63.

43. Andrewes P, Demarini DM, Funasaka K, Wallace K, Lai VWM, Sun H, et al. Do arsenosugars pose a risk to human health? The comparative toxicities of a trivalent and pentavalent arsenosugar. Environ Sci Technol. 2004;38(15):4140-8.

44. Hanaoka K, Goessler W, Ohno H, Irgolic KJ, Kaise T. Formation of toxic arsenical in roasted muscles of marine animals. Appl Organomet Chem [Internet]. 2001;15(1):61-6.

45. Oberoi S, Barchowsky A, Wu F. The global burden of disease for skin, lung, and bladder cancer caused by arsenic in food. Cancer Epidemiol Biomarkers Prev. 2014;23(7):1187-94. Available from: http://cebp.aacrjournals.org/content $/ 23 / 7 / 1187$

46. Martinez VD, Vucic EA, Becker-Santos DD, Gil L, Lam WL. Arsenic exposure and the induction of human cancers. J Toxicol. 2011;2011(431287):1-13.

47. Naujokas MF, Anderson B, Ahsan H, Vasken Aposhian H, Graziano JH, Thompson C, et al. The broad scope of health effects from chronic arsenic exposure: Update on a worldwide public health problem. Environ Health Perspect. 2013;121(3):295-302.

48. Brauner E V, Nordsborg RB, Andersen ZJ, Tjonneland A, Loft S, Raaschou-Nielsen O. Long-term exposure to low-level arsenic in drinking water and diabetes incidence: a prospective study of the diet, cancer and health cohort. Env Heal Perspect. 2014;122(10):1059-65.

49. Yorifuji T, Tsuda T, Doi H, Grandjean P. Cancer excess after arsenic exposure from contaminated milk powder. Environ Health Prev Med. 2011;16(3):164-70.

50. Parvez F, Wasserman GA, Factor-Litvak P, Liu X, Slavkovich V, Siddique AB, et al. Arsenic exposure and motor function 
among children in Bangladesh. Environ Health Perspect. 2011;119(11):1665-70.

51. Lepori ECV. Hidroarsenicismo crónico regional endémico en Argentina. Acta Bioquímica Clínica Latinoam. 2015;49(1):83-104.

52. Mostafa MG, McDonald JC, Cherry NM. Lung cancer and exposure to arsenic in rural Bangladesh. Occup Env Med. 2008;65(11):765-8.

53. Sawada N, Iwasaki M, Inoue M, Takachi R, Sasazuki S, Yamaji T, et al. Dietary arsenic intake and subsequent risk of cancer: The Japan Public Health Centerbased (JPHC) Prospective Study. Cancer Causes Control. 2013;24(7):1403-15.

54. Saint-Jacques N, Parker L, Brown P, Dummer TJ. Arsenic in drinking water and urinary tract cancers: a systematic review of 30 years of epidemiological evidence. Environ Health. 2014;13(1):44. Available from:

55. Lin HJ, Sung TI, Chen CY, Guo HR. Arsenic levels in drinking water and mortality of liver cancer in Taiwan.J Hazard Mater. 2013;(262):1132-8.

56. Perez Carrera A, Perez Gardiner ML, Fernandez Cirelli A. Presencia de arsénico en tejidos de origen bovino en el sudeste de la provincia de Córdoba, Argentina. InVet. 2010;12(1):59-67.

57. Nigra AE, Nachman KE, Love DC, Grau-Perez M, Navas-Acien A. Poultry consumption and arsenic exposure in the U.S. population. Environ Health Perspect. 2017;125(3):370-7.

58. SENASA. Productos registrados. Relación de Productos de Uso Veterinario y Alimentos para Animales con registro vigente. Lima; SENASA; 2013 [Citado el 06 de junio de 2017]. Available from: http://www.senasa.gob.pe/senasa/wp-content/uploads/2014/12/CONSOLIDADO-AL-31-12-2013.pdf

59. Ministerio de Agricultura P. Sectoravícola [Internet]. Lima: Oficina de Estudios Económicos y Estadísticos - OEEE; 2010 [Citado el 06 de junio de 2017]. Available from: http://minagri.gob.pe/portal/ download/pdf/herramientas/boletines/ boletineselectronicos/industriaavicola/2010/Encarte-Sector-Avicola-Junio-17082010.pdf

60. Sun GX, Williams PN, Carey AM, Zhu YG, Deacon C, Raab A, et al. Inorganic arsenic in rice bran and its products are an order of magnitude higher than in bulk grain. Environ Sci Technol. 2008;42(19):7542-6.

61. Raab A, Baskaran C, Feldmann J, Meharg $\mathrm{A}$ a. Cooking rice in a high water to rice ratio reduces inorganic arsenic content. J Environ Monit. 2009;11:41-4.

62. Naito S, Matsumoto E, Shindoh K, Nishimura T. Effects of polishing, cooking, and storing on total arsenic and arsenic species concentrations in rice cultivated in Japan. Food Chem. 2015;168(2015):294-301.

63. Sofuoglu SC, Güzelkaya H, Akgül Ö, Kavcar P, Kurucaovali F, Sofuoglu A. Speciated arsenic concentrations, exposure, and associated health risks for rice and bulgur. Food Chem Toxicol. 2014;64:184-91.

64. Ministerio de Agricultura P. Arroz en el perú [Internet]. Lima: Oficina de Estudios Económicos y Estadísticos - OEEE; 2010. Available from: http://siea.minag.gob.pe/ siea/sites/default/files/2010-ENCARTEARROZ_0.pdf

65. Bundschuh J, Nath B, Bhattacharya P, Liu C-W, Armienta MA, Moreno López M $\mathrm{V}$, et al. Arsenic in the human food chain: the Latin American perspective. Sci Total Environ. 2012;429:92-106.

66. Falandysz J, Rizal LM. Arsenic and its compounds in mushrooms: A review. J Environ Sci Heal Part C. 2016;34(4):217-32.

Correspondencia: María Luisa Medina Pizzali Dirección Facultad de Ciencias de la Salud, Universidad Peruana de Ciencias Aplicadas, UPC, Av. Alameda San Marcos, Chorrillos,

Lima, Perú,

Teléfono: 313-3333 anexo 2710

Correo electrónico:pcnummed@upc.edu.pe

\title{
Ahora nuestra revista incluye:
}

\author{
Publicación anticipada \\ Compartiendo publicaciones científicas con el ciudadano \\ $\checkmark$ Videos de presentaciones conjuntas del Instituto Nacional de \\ Salud y la Academia Nacional de Medicina \\ Galería fotográfica y uideos
}

www.rpmesp.ins.gob.pe 